\title{
A Young Girl with Tuberous Sclerosis Presenting with Recurrent Episodes of Convulsion \& Right Sided Hemiparesis
}

\author{
PARTHA SARATHI SARKER, ${ }^{1}$ SARMIN AKHTER ${ }^{2}$ MD. SAIDUR RAHMAN ${ }^{3}$ MD. MONJURUL KADER \\ CHOWDHURY, ${ }^{1}$ PALASH KUMAR BISWAS, ${ }^{4}$ KHAN ABUL KALAM AZAD ${ }^{5}$
}

\begin{abstract}
:
Tuberous Sclerosis Complex (TSC) is a neurocutaneous syndrome manifested by involvement of multiple system including CNS, kidney, skin, heart, lungs \& eye. It is usually characterized by triad of skin lesions (96\%), seizures (90\%) \& mental retardation (70\%). Most common lesions associated with TSC are angiofibroma of face, ashleaf macules, shagreen patches, subependymal nodules, cortical tubers, mental retardation \&angiomyolipoma of kidney. Here we report a case of a 14 years old girl who presented with recurrent episodes of convulsion, mental retardation \& progressive weakness of right side of body. We investigated the patient to find out the etiology of her illness. After cranial imaging \& other necessary investigations several major \& minor criteria were established, which helped us to reach the final diagnosis.
\end{abstract}

Keywords: Tuberous sclerosis, angiomyolipoma, shagreen patch, subependymal nodules, ashleaf macules, lymphangioleiomyomatosis, seizures.

\section{Introduction:}

Tuberous Sclerosis (also known as Tuberous Sclerosis Complex-TSC) is a multisystem genetic disorder affecting cellular differentiation, proliferation \& migration early in development. It results in a variety of hamartomatous lesions to grow predominantly in the brain \& also in other vital organs of the body such as the kidneys, heart, eyes, lungs \& skin. ${ }^{1}$ In 1880 , a French physician- Bourneville first described the cerebral manifestations of this disorder applying the term 'sclerose (hard) tubereuse (swelling)' to indicate the superficial resemblance of the lesion to a potato. The cortical manifestations may sometimes still be known by the eponym "Bourneville's Disease". In 1908,Vogt set forth the triad of intractable epilepsy, mental retardation \& adenoma sebaceum; this description represented the hallmark of TSC to most clinicians. But, less than one-third of affected persons fit the classic constellation of symptoms. ${ }^{2}$

TSC can present at any age $\&$ both sexes are equally affected. In infants \& children it usually is identified as a cause of epilepsy, autism or cardiac failure. Older persons may present

1. Indoor Medical Officer, Medicine unit-1,Dhaka Medical College Hospital, Dhaka.

2. Post-graduate Trainee Doctor, Medicine unit-1, Dhaka Medical College Hospital, Dhaka.

3. Assistant Registrar, Medicine unit-1, Dhaka Medical College Hospital, Dhaka.

4. Registrar, Medicine unit-1, Dhaka Medical College Hospital, Dhaka.

5. Professor \& Head, Dept.of Medicine, Dhaka Medical College, Dhaka.

Corresponding author: Dr. Partha Sarathi Sarker, Indoor Medical Officer, Dept. of Medicine, Dhaka Medical College Hospital, Dhaka.Email: parthadmc60@yahoo.com. with renal failure or pulmonary or cutaneous manifestations in absence of prominent or any neurological symptoms. Pulmonary disease like lymphangiomyomatosis occurs predominantly in women of $3^{\text {rd }} \& 4^{\text {th }}$ decades of life. Some studies suggested that males are more likely to suffer neurological morbidity,but this has not been demonstrated conclusively. So far, Tuberous Sclerosis has been found to affect all races without any clear-cut predominance. ${ }^{3}$

This disorder affects as many as 25000-40000 individuals in the United States \& about 1-2 milion individuals worldwide, with an estimated birth incidence of 1 case per 6000 population \& prevalence of 1 case per 10000 population. ${ }^{3}$ In Bangladesh, only a few (only 2) cases of TSC were reported to date. Here, we will report a case of Tuberous Sclerosis presented with intractable seizure disorder, mental retardation \& hypopigmented skin lesion.

\section{Case report:}

A 14 year old muslim female got admitted at Medicine department of DMCH on $3^{\text {rd }}$ April 2016 with the complaints of recurrent episodes of convulsion for 9 years and progressive weakness of right side of the body for 3 months. Convulsion started at 6 years of her age which was generalized, persisting 1-2 minutes at each episode, occuring 2-3 times daily, sometimes at an interval of 2-3 days interval. Those attacks of convulsion were associated with salivation, rolling of eyeball \& post ictal confusion for 30 minutes, but not associated with any urinary or faecal incontinence. With these complaints she was treated by local quacks. One and a half year back she was admitted in DMCH with recurrent episodes of convulsion and was diagnosed as a case of primary generalized epilepsy on the basis of EEG and CT 
scan of head and was discharged with antiepileptic drugs. After that she was seizure free for next 6 months with regular anti-epileptic medications. At one point of time, she stopped taking all the medications \& afterwards she started having convulsions which were of similar character as it occurred in the past. But she did not restart the treatment. Rather, after 7 months of that time, she developed high grade continued fever without any physical symptom to guide to the etiology of the illness. 2 days later of initiation of fever, she became disoriented with irrelevant talk and squint in her right eye. After 6-7 days, along with subsidence of temperature her level of orientation, speech \& squint also improved. During this interval of time, convulsion occurred at previous manner \& frequency as stated above. At the month of January, 2016 she developed progressive weakness of right side of the body,initially it was mild in severity \& she was able to walk with some difficulties but gradually weakness became severe $\&$ she cannot walk properly without any support for last 1 month. On query, she also gave no history of joint pain, oral ulceration, alopecia, dysphagia, respiratory difficulty, trauma, bowel or bladder disturbance $\&$ family history of such type of illness. She was born with full term pregnancy by normal vaginal delivery at home. Her developmental milestones were normal. She was not immunized according to EPI schedule. Her age of menarche was 13 years \& menstruation is irregular.There is no consanguity of marriage of her parents. She belongs to lower middle class family, lives in tin shed kacha house, drinks tube well water and uses sanitary latrine. She has 4 brothers $\&$ all family members are enjoying good health. Her school performance was poor for which she stopped going to school when she was studying at class Six.

On examination, she was well behaved, co-operative, with average body built. Pulse rate was 72 beats/min \& blood pressure was 130/90 mmhg. There was no lymphadenopathy or thyromegaly, secondary sexual characteristic were well developed There was a hypopigmented non-pruritic macule on lower back which is $3 \times 1.5 \mathrm{~cm}$ in size, sensation was intact over the hypopigmented area.On Examination of nervous system, higher psychic function was normal except slow retarded speech, MMSE score was 24 out of 30 , all cranial nerves were intact,Fundoscopic examination was normal, no retinal hamartoma or any other abnormality was found. On examination of motor system: bulk of muscle was normal, tonec increased in right side, power of right side of the body was $3 / 5$, all reflexes in right side were exaggerated, clonus was absent, plantar response was extensor on right side, gait was of spastic variety sensory system was intact, cerebellar function test was normal, signs of meningeal irritation were absent. All other systems revealed no abnormality. Investigation revealed $\mathrm{CBC}-\mathrm{Hb} \% 13.5 \mathrm{gm} / \mathrm{dl}$, $\mathrm{TC}$ of WBC 9010/cubic mm \& TPC 2,55,000/cubic mm of blood, ESR$41 \mathrm{~mm}$ in $1^{\text {st }}$ hour, ANA screening was negative, Antiphospholipid Ab was negative,Serum calcium level was 9.2 $\mathrm{mg} / \mathrm{dl}$, PTH level was $19.6 \mathrm{pg} / \mathrm{ml}$, CPK level-30u/L, ECGnormal, Echocardiography- a large heterogenous mass in LV apex suggestive intracardiactumour-Rhabdomyoma with an EF of $68 \%$. USG of whole abdomen was normal,CT scan of chest was normal, CT scan of brain revealed multifocal calcified sub-ependymal nodules along both lateral ventricles suggestive of tuberous sclerosis with bilateral deep white matter ischaemic changes(Lt.>Rt.) with mass effect. MRI of brain revealed- tiny subependymal signal void areas noted along the outer wall of right lateral ventricle corresponding to the CT-detected calcified nodules \& intense gyral enhancement in both frontal lobes \& left parietal lobe with profuse perilesional edema resulting in mass effect \& causing ipsilateral lateral ventricle compression as well as contralateral midline shifting. These findings were suggestive of tuberous sclerosis with evidence of laminar cortical necrosis. EEG was normal. Under Wood's light examination the hypopigmented patch revealed ash-leaf hypomelanotic
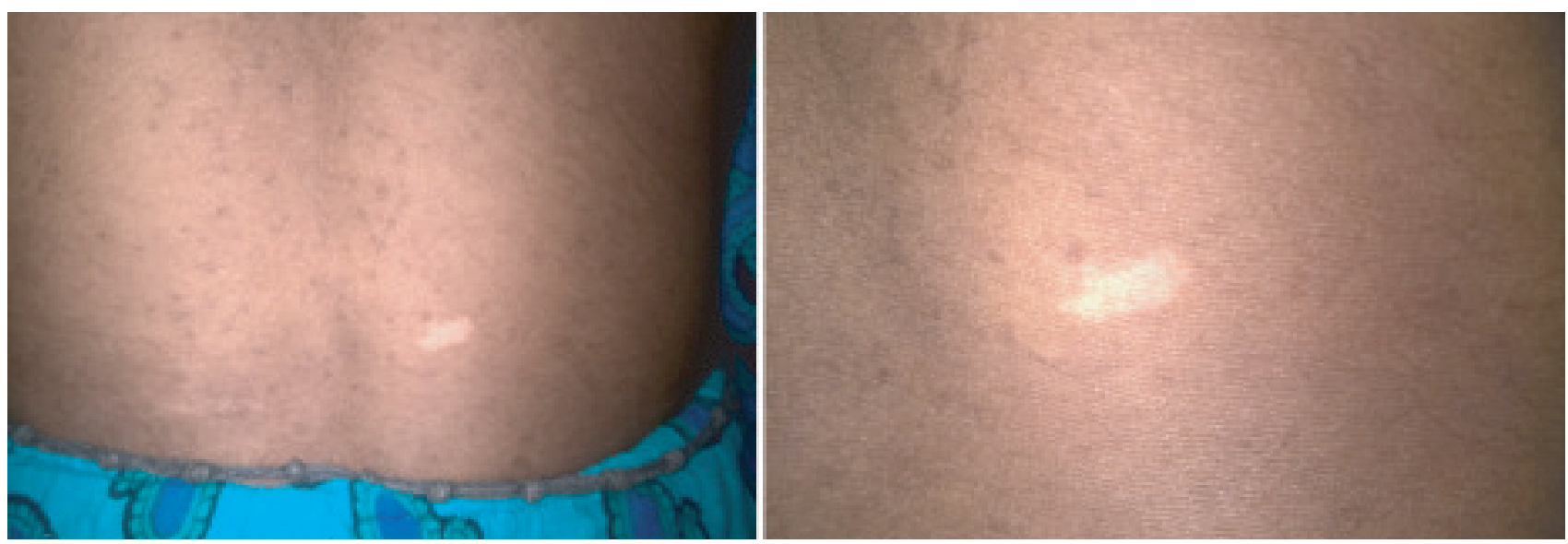

Fig.-1: Ash-leaf hypomelanotic macule on lower back 


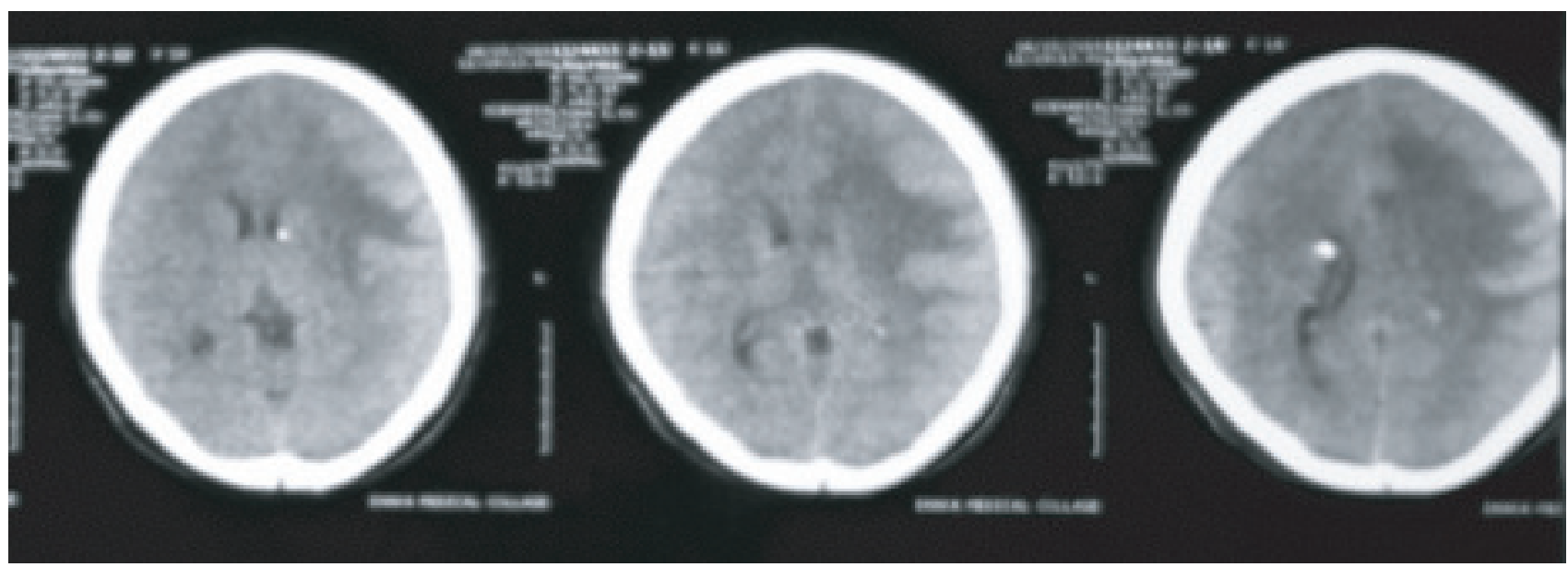

Fig.-2: CT scan of brain
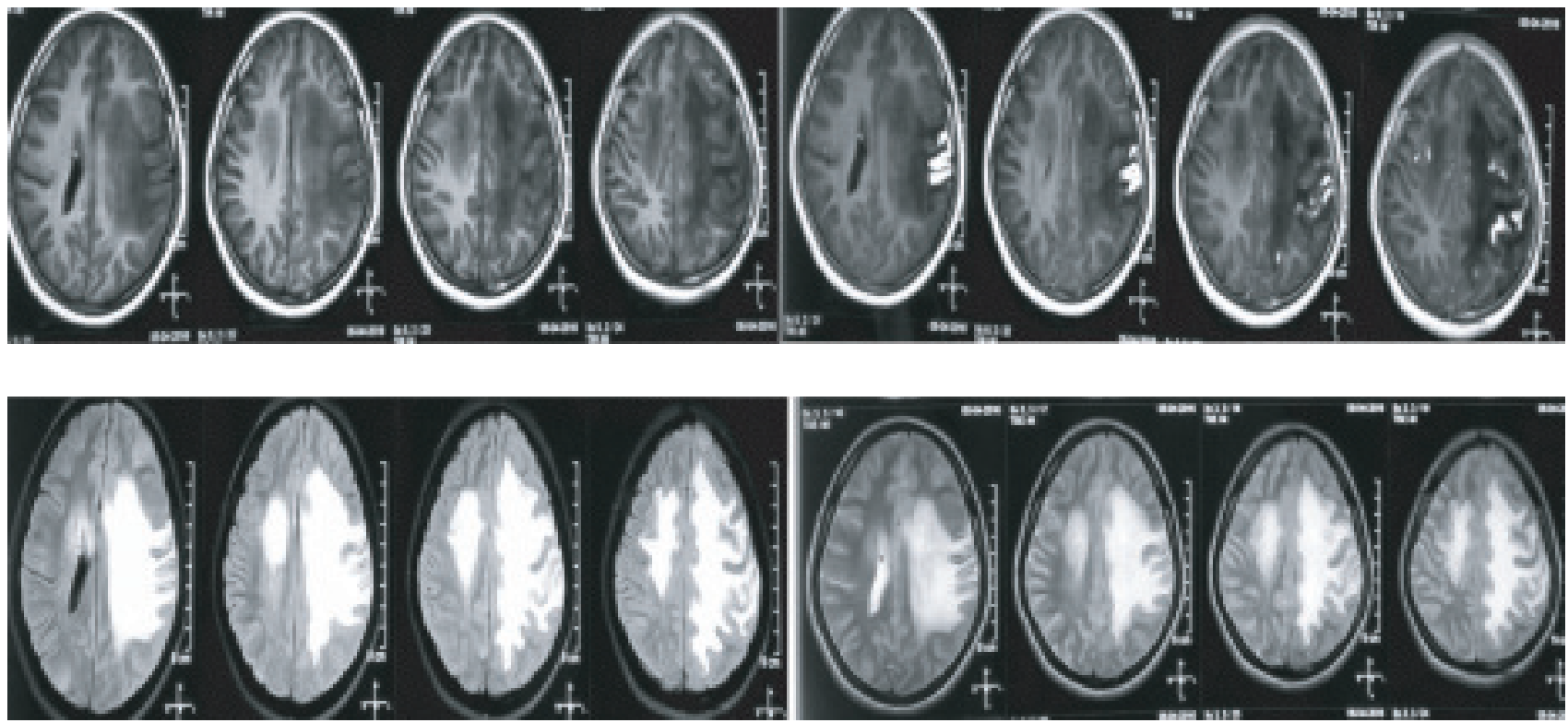

Fig.-3: MRI of brain- T1, T1 with contrast, FLAIR \& T2 image.

macule,colonoscopic finding was normal. Taken into consideration of the radiological findings of CT scan \& MRI of brain, cardiac rhabdomyoma on echocardiography \& ashleaf macule under wood's light examination, the patient was diagnosed as a case of Tuberous Sclerosis.

The patient was treated with Inj. Methylprednisolone $1 \mathrm{gm}$ daily for 3 days followed by oral prednisolone for next 1 month \& were planned for repetition of MRI of brain after completion of oral steroid. Convulsion was managed with sodium valproate \& Phenytoin. For right sided weakness she was given physiotherapy for a few days. With this treatment, her convulsion was well controlled \& her right sided weakness also improved. For cardiac lesion, she was advised to consult with cardiac surgery later on. The patient was managed in collaboration with the departments of Cardiology, Neurology, Neurosurgery, Dermatology\& Physical Medicine.

\section{Discussion:}

Tuberous Sclerosis Complex is a genetic disorder caused by defects or mutations on two genes- TSC1 and TSC2. Only one of the genes needs to be affected for TSC to be present. The TSC1 gene, discovered in 1997, is on chromosome 9 \& produces a protein called hamartin. The TSC2 gene, discovered in 1993, is on chromosome 16 \& produces the protein tuberin. These proteins act as tumor growth suppressors, agents that regulate cell proliferation \& 
differentiation ${ }^{3}$. Some individuals may inherit the disorder from a parent with TSC, in which case it shows an autosomal dominant inheritance. Rarely, individuals may also acquire tuberous sclerosis through a process called gonadal mosaicism. ${ }^{4}$

Patients may present with a combination of symptoms including seizures, intellectual disability, developmental delay, behavioral problems, skin abnormalities \& lung \& kidney diseases. Particular symptoms occur at various points in the life span.

According to the Diagnostic Criteria Committee of the National Tuberous Sclerosis Association (USA): ${ }^{5}$

Major features of TSC include the followings-

- Facial angiofibromas or forehead plaque

- Nontraumatic ungual or periungual fibroma

- Hypomelanotic macules (>3)-to be confirmed by wood's light or UV light.

- Shagreen patch (connective tissue nevus)

- Multiple retinal nodular hamartoma

- Cortical tubers.

- Subependymal nodule

- Subependymal giant cell astrocytoma

- Cardiac rhabdomyoma, single or multiple

- Lymphangioleiomyomatosis.

- Renalangiomyolipoma.

Minor features of TSC include the following:

- Multiple randomly distributed pits in dental enamel.

- Hamartomatous rectal polyps: Histologic confirmation is suggested.

- Bone cysts: Radiographic confirmation is sufficient.

- Non calcified subependymal nodules.

- Gingival fibromas

- Nonrenalhamartoma: Histologic confirmation is suggested.

- Retinal achromic patch

- "Confetti" skin lesions

- Multiple renal cysts

The following are the diagnostic criteria for TSC: 5

- Definite TSC - Two major features or one major feature plus two or more minor features

- Possible TSC - Either one major feature or two or more minor features

Diagnosis should be possible in most cases using established clinical criteria. Molecular genetic testing is useful in uncertain or questionable cases, for pre-natal diagnosis \& for screening family members of an affected individual. Under optimal circumstances, genetic testing identifies mutations in upto $75-80 \%$ of affected individuals. Therefore a negative genetic diagnostic test result does not exclude the diagnosis. But the utility of molecular testing is limited by the cost.

There is no complete cure of Tuberous Sclerosis, although treatment is available for a number of symptoms. Antiepileptic medications are the main stay of therapy for patients with TSC. The choice of AED for treating seizures is based on patient's seizure type, age of the patient, AED side effect profile \& formulations available. Vigabatrin is the drug of first choice for children with TSC \& infantile spasm. Topiramate, lamotrigine\& valproateare also useful. Carbamazepine, oxcarbazepine\& phenytoin are often valuable in older children $\&$ adults, in whom partial seizures predominate. The USFDA has approved the drug everolimus to treat sub-ependymal giant cell astrocytoma (SEGA) \& angiomyolipoma kidney tumours. Some surgical options such as focal cortical resection, corpus callostomy \& vagus nerve stimulation are also available. ${ }^{5}$ In addition, SEGAs require resection if they produce hydrocephalus or significant mass effect. Intervention programs including special schooling \& occupational therapy may benefit individuals with special needs \& developmental issues. In most cases, cardiac tumours will not need treatment. In some rare cases, surgery may be required to remove the tumors if they begin to seriously affect the function of the heart. Rapamycin or Sirolimus may be effective to shrink the lung tumours, but if tumours lead to a collapsed lung, emergency surgery may be required to repair the lung. 6

\section{Conclusion:}

The prognosis for individuals with TSC is highly variable and depends on the severity of symptoms. Those individuals with mild symptoms usually do well and have a normal life expectancy.Individuals who are severely affected can suffer from severe mental retardation and persistent epilepsy.

Earlier age of seizure commencement ( $<6$ months) is associated with poor seizure outcome and poor intellectual capabilities. Infantile spasms and severely epileptogenic EEG patterns are related to the poor seizure outcome, poor intellectual capabilities and autistic behavior. Higher tubers numbers is associated with poor seizure outcome and autistic behavior. Left-sided tuber burden is also associated with poor intellect. So, close follow up for the mental development and early control of seizures are recommended to reduce the risk factors of poor outcome. Also early diagnosis of autism will allow for earlier treatment and the potential for better outcome for children with TSC. ${ }^{7}$ 
All individuals with TSC are at risk for life-threatening conditions related to the brain tumors, kidney lesions, or LAM.Continued monitoring by a physician experienced with TSC is important. With appropriate medical care, most individuals with the disorder can look forward to normal life expectancy.

\section{Conflict of interest: None}

\section{References:}

1. Tuberous Sclerosis Fact Sheet. NINDS: 2006-04-11. Modified 2016-01-21.

2. Haque s, Morshed T, Begum H, Islam S, Fatema K, Begum A, Shoyab M. TuberousSclerosis Complex: A Case Report. ChattagramMaa-o-Shishu Hospital Medical College Journal. 2014;13(3):89-92.
3. Ownes J, Bodensteiner JB. Tuberous Sclerosis Complex: Genetics, Clinical Features \& Diagnosis. UpToDate:updated 2015-06-16.

4. CrinoPB, Nathanson KL, Henske EP. The tuberous sclerosis complex. N Engl J Med 2006;355:1345-48.

5. Franz DN, Thomas CW. Tuberous Sclerosis. Medscape: updated 2015-10-14.

6. Ishii M, Asano K, Kamishi N, Hayashi y. Tuberous Sclerosis diagnosed by incidental CT findings multifocal micronodularpneumocyte hyperplasia: a case report. Journal of medical case reports 2012;6:352-56.

7. Zarei M, Collins VP, Chandran S, Valler D, Higgins JNP, Compston DAS. Tuberous Sclerosis present in late adult life. J NeurolNeurosurg Psychiatry 2002;73:436-40. 